\title{
Heterogeneous Wireless Sensor Networks for Flood Prediction Decision Support Systems
}

\author{
Karl Andersson \\ Pervasive and Mobile Computing Laboratory \\ Luleå University of Technology \\ SE-931 87 Skellefteå, Sweden \\ karl.andersson@1tu.se
}

\author{
Mohammad Shahadat Hossain \\ Department of Computer Science and Engineering \\ University of Chittagong \\ University-4331, Bangladesh \\ hossain_ms@cu.ac.bd
}

\begin{abstract}
Recent advancements in the fields of sensor equipment and wireless sensor networks have opened the window of opportunity for many innovative applications. In this paper, we propose a new architecture for building decision support systems using heterogeneous wireless sensor networks. The architecture is built around standard hardware and existing wireless sensor networks technology. We show the effectiveness of the proposed architecture by applying it to a flood prediction scenario.
\end{abstract}

Keywords__ Internet of Things; Wireless Sensor Networks; Flood prediction; Belief-rule-based Decision Support Systems

\section{INTRODUCTION}

The Internet of Things (IoT) [1] revolution where cost efficient low-powered sensors and actuators are being deployed in massive scale over large areas and communicating over the Internet is now becoming a reality. In combination with stateof-the-art wireless sensor networks (WSNs) [2] technologies interesting applications can easily be developed reaching far beyond what has been delivered by M2M (Machine-tomachine) systems until now. This way, 50 billion devices are predicted to be connected in 2020 [3].

Applications include a variety of examples making objects smarter like smart cities, smart regions, smart grids, smart homes, and home automation, Earlier, such systems were typically designed in stovepipes where end nodes where very much tied to a specific communication technology and tailor made backend systems. The main trend in both research and commercial applications is the move from vendor-specific installations to standards based and open source implementations.

This paper proposes a new architecture for building decision support systems using heterogeneous wireless sensor networks where the architecture is built around standard hardware and existing wireless sensor networks technology. We show the effectiveness of the proposed architecture by applying it to a flood prediction scenario.

The rest of the paper is organized in the following way. Section II covers related work, while Section III outlines our architecture proposal. Section IV describes the flood prediction scenario, while Section V finally concludes our findings.

\section{RELATED WORK}

Flood prediction systems have been used in many parts of the world since long ago. With the introduction of WSN technology such systems have been easier to design for higher accuracy and faster reactions.

Ahmad et al. [4] made a reviewed flood prediction and disaster risk analysis using of GIS-based wireless sensor networks. Furthermore, Seal et al. [5] described and evaluated a simple flood-forecasting scheme using wireless sensor networks, while Khedo [6] described real-time flood monitoring using wireless sensor networks. Moreover, Basha et al. [7] proposed and evaluated model-based monitoring for early warning flood detection, while Shukla et al. [8] described how to design an architectural model for flood monitoring using wireless sensor networks. An interesting case from Brazil was described by Furquim et al. [9] where an accurate floodforecasting model using WSNs and chaos theory was studied in a real deployment. Finally, Ishida et al. [10] proposed a realtime disaster damage information sharing system for disaster countermeasures headquarters at the time of large-scale natural disaster.

Our previous work includes [11] where we described how smart risk assessment systems can be built using belief-rule based decision support systems and WSN technologies and [12] where a secure and scalable system for sharing information in smart homes was described and evaluated.

\section{PRoposed ARChItecture}

\section{A. Challenges and Architecture Requirements}

When designing a suitable architecture supporting data collection from low-cost sensors spread over a large geography, we considered that many existing solutions are non-interoperable and vendor-specific. Our proposed solution is standards-based and addressing the following basic requirements:

- Modularity (separating functionality within different entities with clearly defined interfaces);

- Scalability (enabling an architecture that scales with an increased number of connected nodes); and 
- Extendibility (enabling step-wise deployment and adding/removing of capabilities at later stages)

Our proposed architecture is composed of three distinct parts, namely:

- Cloud/Server-side entity supporting processing managing, monitoring data from IoT devices;

- Suitable networking model to handle sensor communication; and

- Suitable devices (low-cost, long lifetime, easy deployable, etc.)

The architecture follows a layered structure separating the above-mentioned functions also taking security and mobility into consideration.

\section{B. Proposed Heterogeneous WSN Architecture}

To support the challenges and requirements laid out in the previous subsection, we designed a heterogeneous wireless sensor network (WSN) architecture with three distinct layers, see figure 1 .

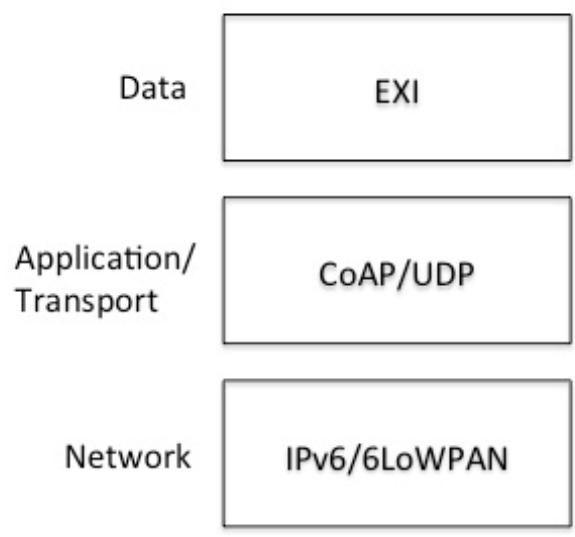

Fig. 1. Layered architecture.

The data layer is responsible for handling of data and our choice is to use the Efficient XML Interchange (EXI), being essentially an encoding format that allows efficient interchange of the an XML information set. EXI also illustrates effective processor implementations of that encoding.

For the application and transport layer, where a basic connectivity service is established our choice is the Constrained Application Protocol (CoAP) on top of User Datagram Protocol (UDP). CoAP is a REST-ful application protocol modeled on HTTP semantics with a much smaller footprint than MQTT or HTTP itself by taking a binary rather than text-based approach.

The network layer in our solution is realized as an IPv6 network with support for 6LOWPAN (IPv6 over Low power Wireless Personal Area Networks). This is the state-of-the-art technology for enabling IP to low power devices like sensors cutting down packet overhead, allowing for more payload data to be transported. Also, 6LOWPAN only uses $30 \mathrm{~KB}$ for a full featured stack compared to $90 \mathrm{~KB}$ for ZIGBEE.

\section{Communication solutions and sensor hardware for the specific scenario}

To support the afore-mentioned challenges and requirements we are using Arduino, an open-source electronics platform based on easy-to-use hardware and software. Sensors are measuring rainfall, river water level, and river water flow. For the actual communication we are proposing a combination of Zigbee, Ethernet, Wi-Fi connectivity and SMS. In combination with that, we propose a sensor data processing system converting analog data in a form that could be fed into the BRB ES. Last, but not least, we also propose a calibration algorithm for sensor data.

\section{FLOOD PREDICTION SCENARIO}

The data of the various flood intensifying factors as mentioned in the previous section is generated by the sensor data processing system in a format that can be fed into the Belief Rule Based Expert System (BRBES) consisting of a knowledge base and an inference engine. Belief Rule Base has been used to build the knowledge base while Evidential Reasoning, which is multi criterion decision analysis procedures and can handle both qualitative and quantitative data, considered as the inference engine [13]. Figure 2 illustrates the interface of the BRBES. Various flood intensifying factors can be categorized as meteorological (X8), geological (X9), river characteristics (X10), topographical (X11) and human activities (X12). These are shown in the middle level of the BRB tree as shown in figure 1. The middle level factors can be termed as drivers. The value of each driver depends on a number of factors. For example, meteorological driver depends on rainfall (X22) and rainfall duration (X23). These factors (X22, X23) are shown as the leaf nodes of the $\mathrm{BRB}$ tree.

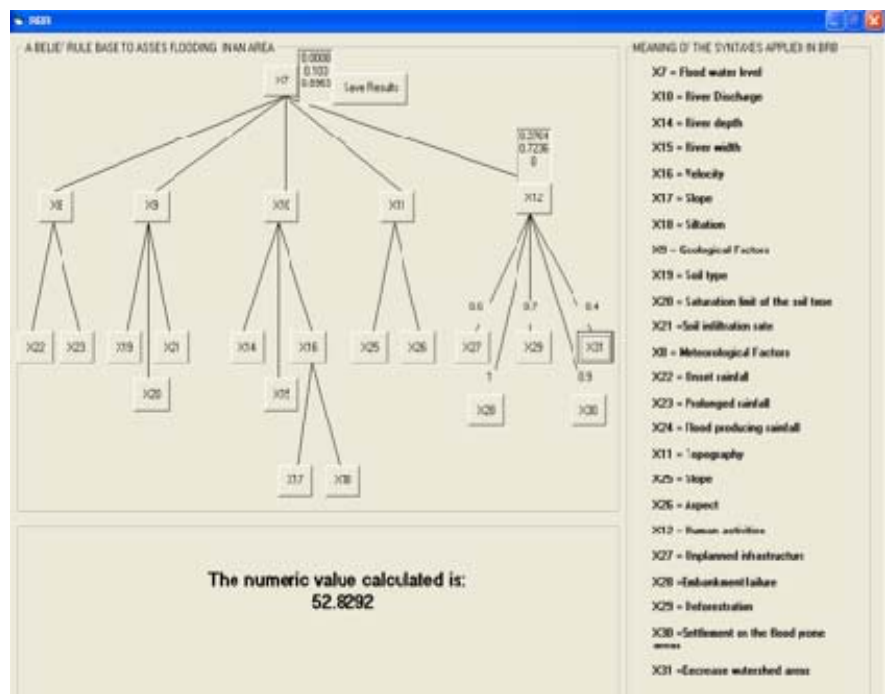

Fig. 2. BRBES's Interface.

Other leaf node factors consist of soil infiltration rate, soil saturation limit, soil type, river depth, slope, aspect, unplanned infrastructure etc. as can be seen from figure 1. The input data includes the collection of the data related to the leaf node factors of the BRB tree. It is interesting to note that some of 
these leaf node data are quantitative in nature and others are qualitative in nature. For example, rainfall data is an example of quantitative data while unplanned infrastructure is an example of qualitative data. Sensors are suitable to collect the quantitative data and hence, various sensors have been used to collect the data, which are quantitative in nature. However, qualitative data has been collected by looking at the documents and human perception of a flood prone area. Literature showed that various flood prediction models developed either using qualitative data or using the quantitative data but not by considering the both types of data [14][15]. In addition, various types of uncertainty such as ignorance, incompleteness, randomness, and vagueness, imprecision exist with these data [16][17].

There exists various knowledge representation schemas such as Propositional Logic (PL), First Order Logic (FOL), Fuzzy Logic (FL), Semantic Nets, Frames, Case based reasoning but they are not equipped to handle the mentioned types of uncertainty [18][19]. For example, FL can handle uncertainty due to vagueness or imprecision but it cannot handle uncertainty due to ignorance or incompleteness or ignorance in fuzziness. PL and FOL can only handle assertive knowledge, which is either true or false. Hence, it is necessary to have a knowledge representation schema, which can handle the above uncertainties. Belief Rule Base, which is a knowledge representation schema, can handle the mentioned type of uncertainties. There exits inference mechanism such as Forward Chaining and Backward chaining but they are not equipped to process uncertain data [20][21]. Therefore, ER has been considered as an inference engine, which is used to aggregate the rules to obtain the value of the consequent of a BRB.

The Belief Rule Base (BRB) is an extension of traditional IF-THEN rule base. A belief rule has antecedent part and consequent part. Antecedent attribute take referential values and possible belief degrees are associated with each consequent. The rule weights, antecedent attribute weight, and belief degrees are knowledge representation parameters used in BRB to capture the uncertainty. A belief rule can be defined as:

\section{Rk:IF Rainfall is Medium AND Rainfall Duration is High THEN Meteorological Condition is $\{($ Severe, 0$)$, (Moderate, 0.4$),($ Low, 0.6$)\}$}

In the above rule 'Rainfall' and 'Rainfall Duration' are the antecedent attributes, while 'Medium' and 'High' are the referential values. 'Meteorological Condition' is the consequent attribute with referential values such as 'severe', 'moderate', and 'low'. This rule is complete because the summation of degree of belief associated with each referential value of the consequent attribute is one. If the summation is less than one then the rule is considered as incomplete, which may due to incomplete information or ignorance. The relationship between antecedent attributes and the consequent attribute is non-linear, which is linear in case of IF-THEN rule. There could be $L$ number of rules in a BRB $(K=1, \ldots, L)$. Each antecedent and consequent attributes may have $N$ number of referential values.

The inference procedures consists various steps including input transformation, rule activation, belief update and rule aggregation using Evidential Reasoning approach. Figure 3 shows the structure of the inference procedures of the BRBES.

The task of input transformation consists of distributing the input data over the referential values of the attribute of a rule, which called matching degree. In our case, when sensor data for a particular flood intensifying factor is received from the sensor it is then distributed over the referential values of the antecedent attribute of a rule. For example, an antecedent attribute is "Rainfall" and its referential values are "High", Medium" and "Low". A belief rule base consists of too many rules and the number of rules in a rule base depends upon the antecedent attributes and their referential values. For example, it can be observed from Figure 2 that 'Meteorological Condition" sub rule base has two antecedent attributes namely "rainfall" and "rainfall duration" and if each of the attributes has three referential values then this sub rule base consists of nine rules.

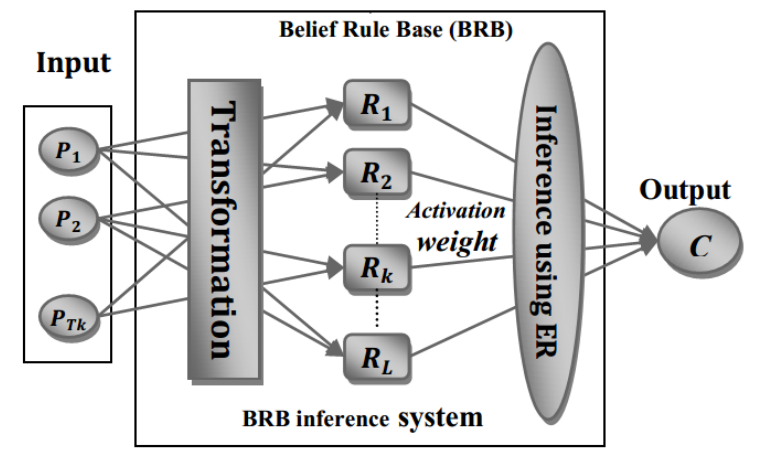

Fig. 3. Single-layer BRB inference architecture with RIMER Methodology.

Once the matching degree is calculated the rules are called packet antecedent or they are active or they reside in the short term memory while the rule base resides in the long term memory. The calculated matching degree is used to calculate the activation weight of each rule. It is interesting to note that each rule does not have the same weight in calculating the referential values of the consequent attribute. The summation of the rule activation weight of a rule base should need to be one.

When an input data for any of the leaf nodes is ignored then the belief degree associated with each rule in the rule base should need to be updated. This is done by following the procedures mentioned in [13]. The aggregation of the rules is carried out by using either analytical or recursive evidential reasoning algorithm [13]. By applying the ER algorithm the rules of the "Human Activity" sub rule base have been aggregated by taking account of the input data as shown in Figure 2. The combined degree of belief associated with the referential values (high, medium, low) of the consequent attribute (human activity) of this sub rule base obtained as $\{($ High, 0.2764), (Medium, 0.7736), (Low, 0)\}, which can be seen from Figure 2. The combined degree of belief, associated with the referential values of the flood water level (X7), obtained which is $\{($ High, 0.0008), (Medium, 0.103), (Low, $0.8963)\}$. The fuzzy value can be converted into crisp value by using the utility score associated with each referential value to obtain flood water depth, which is in this case is $52.8292 \mathrm{~cm}$. 


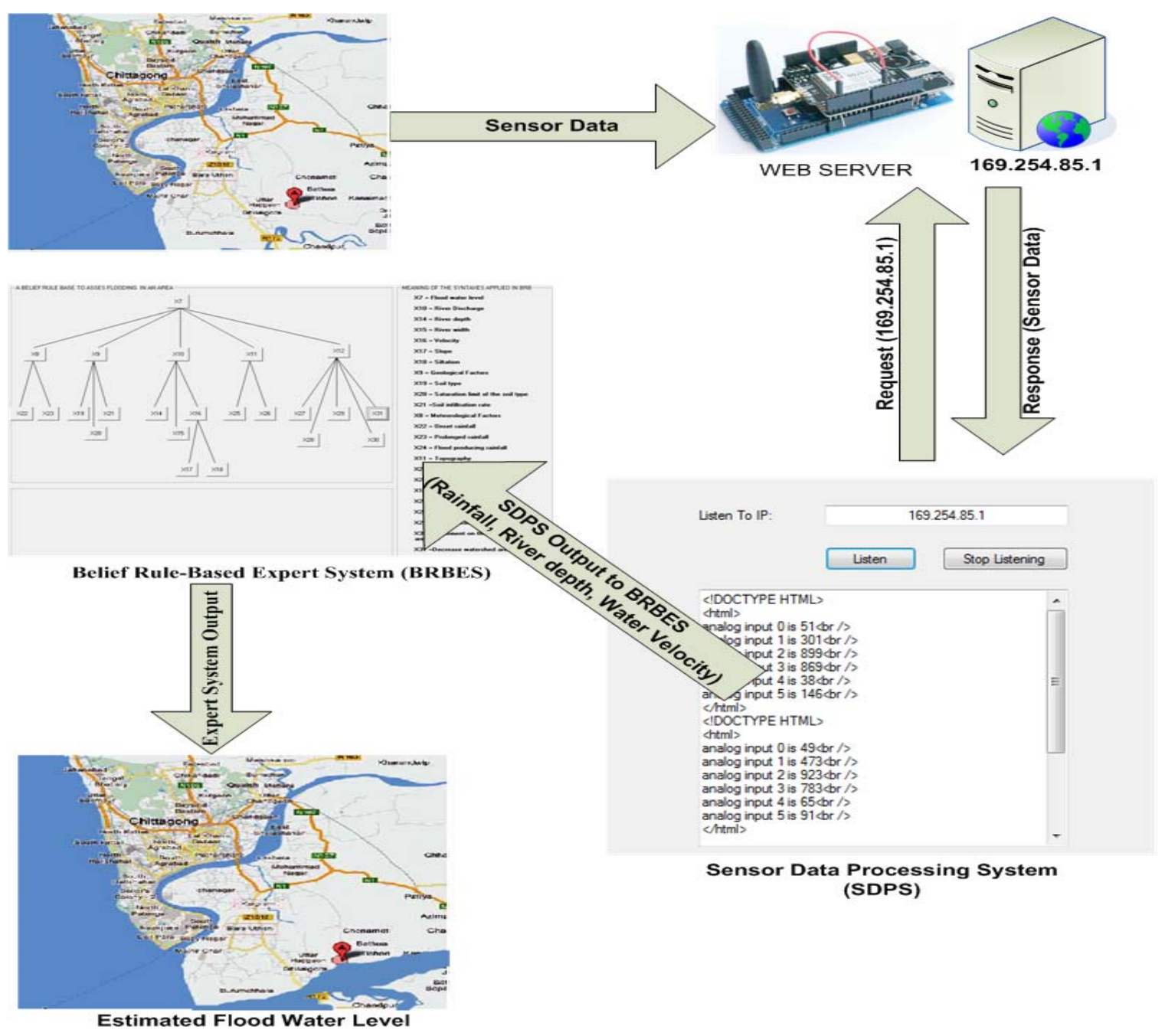

Fig. 4. Setup of the flood prediction scenario.

In this way, BRBES allows the generation of different flood scenarios of an area by taking account of sensor data in real time basis. Consequently, various decisions or response strategies can be developed to reduce the risks of flooding in an area.

\section{DISCUSSION}

The architecture proposed in Section 3 is being used in flood prediction scenario as described in Section 4. We are using the setup depicted in Figure 4. Data is visualized in GIS system showing values in different regions on an interactive map.

The proposed architecture has shown to successfully meet the challenges and requirements outlined. We are now developing new scenarios and collecting data from the system developed.

\section{ACKNOWLEDGMENT}

This work has been supported by the Swedish Research Council under grant 2014-4251.

\section{REFERENCES}

[1] L. Atzori, A. Iera, \& G. Morabito, "The Internet of Things: A survey," Wiley, in Computer Networks 54(15):2787-2805, Oct. 2010.

[2] I. Akyildiz \& M. Can Vuran, "Wireless Sensor Networks," Wiley, Singapore, 2010.

[3] Cisco, 2010. Available at http://share.cisco.com/internet-of-things.html on December 31, 2014.

[4] N. Ahmad, M. Hussain, N. Riaz, F. Subhani, S. Haider, K. Alamgir, \& F. Shinwari, "Flood Prediction and Disaster Risk Analysis using GIS based Wireless SensorNetworks, A Review," in Journal of Basic and Applied Scientific Research 3(8):632-643, 2013.

[5] V. Seal, A. Raha, S. Maity, S. Mitra, A. Mukherjee, \& M.Naskar, "A Simple Flood Forecasting Scheme Using Wireless Sensor Networks," in International Journal of Ad hoc, Sensor \& Ubiquitous Computing 3(1):45-60, Feb. 2012.

[6] K. Khedo, "Real-Time Flood Monitoring Using Wireless Sensor Networks," in The Journal of the Institution of Engineers Mauritius 2013:59-69, Sep. 2013.

[7] E. Basha, S. Ravela, \& D. Rus, "Model-Based Monitoring for Early Warning Flood Detection," in Proceedings of the 6th ACM conference on Embedded network sensor systems (SenSys'08), Raleigh, NC, USA, Nov. 2008. 
[8] S. Shukla \& G. Pandey, "To design an Architectural Model for Flood Monitoring using Wireless Sensor Network System," in International Journal of Computer Science and Information Technologies 5(1):502507, 2014.

[9] G. Furquim, R. Mello, G. Pessin, B. Faiçal, E. Mendiondo, \& J. Ueyama, "An Accurate Flood Forecasting Model Using Wireless Sensor Networks and Chaos Theory: A Case Study with Real WSN Deployment in Brazil," in Proceedings of 15th International Conference, EANN 2014, Sofia, Bulgaria, Sep. 2014.

[10] T. Ishida, K. Takahagi, A. Sakuraba, N. Uchida, \& Y. Shibata, "The Real-time Disaster Damage Information Sharing System for Information Acquiring in Large-scale Natural Disaster," in Journal of Internet Services and Information Security 4(3):40-58, Aug. 2014.

[11] K. Andersson \& M. S. Hossain, "Smart risk assessment systems using belief-rule-based DSS and WSN technologies," in Proceedings of 2014 4th International Conference onWireless Communications, Vehicular Technology, Information Theory and Aerospace \& Electronic Systems (VITAE), Aalborg, Denmark, May 2014.

[12] R. Ul Islam, M. Schmidt, H. Kolbe, \& K. Andersson, "Secure and Scalable Multimedia Sharing between Smart Homes," in Journal of Wireless Mobile Networks, Ubiquitous Computing, and Dependable Applications 5(3):79-93, Sep. 2014.

[13] J. Yang, J. Liu, J. Wang, \& H. Sii, H. Wang, “A belief rule-base inference methodology using the evidential reasoning approach RIMER," in IEEE Transactions on Systems, Man, and Cybernetics Part A: Systems and Humans 36(2):266-285, March 2006.

[14] J. Bouma, D. Franc ois, \& P. Troch, "Risk assessment and water management," in Environmental Modelling \& Software 20(2):141-151, Feb. 2005.
[15] S. van Manen \& M. Brinkhuis, "Quantitative flood risk assessment for Polders," in Reliability Engineering \& System Safety 90(2-3):229-237, Nov.-Dec. 2005.

[16] J. Hall, E. Evans, E. Penning-Rowsell, P. Sayers, C. Thorne, \& A. Saul, "Quantified scenarios analysis of drivers and impacts of changing flood risk in England and Wales: 2030-2100," in Global Environmental Change Part B: Environmental Hazards 5(3-4):51-65, 2003.

[17] R. J. Nicholls, "An Expert Assessment of Storm Surge "Hotspots"”. Final Report, Centre for Hazard and Risk research, Lamont-Doherty Observatory, Columbia University, 2003.

[18] C. Angulo, J. Cabestany, P. Rodríguez, M. Batlle, A. González, \& S. de Campos, "Fuzzy expert system for the detection of episodes of poor water quality through continuous measurement," in Expert Systems with Applications 39(1):1011-1020, 2012.

[19] T. Liu, J. Singonahalli, \& N. Iyer, "Detection of roller bearing defects using expert system and fuzzy logic," in Mechanical Systems and Signal Processing 10(5):595-614, 1996.

[20] S. Rahman \& M. S. Hossain, "A Belief Rule Based Clinical Decision Support System to Assess Suspicion of Heart Failure from Signs, Symptoms and Risk Factors," in Proceedings of 2nd International Conference on Informatics, Electronics \& Vision, May, 2013, Dhaka, Bangladesh, Print ISBN: 978-1-4799-0397-9, INSPEC Accession Number: 13684176.

[21] M. S. Hossain, S. Akter, \& S. Rahman, "A Belief Rule Based Expert System to Assess Meditation," in Proceedings of the the 2014 International Conference on Computational Science and Computational Intelligence conference, March 10-13, 2014, Las Vegas, NV, USA. 